\title{
Einblicke in das italienische Erbrecht - allgemeine Grundsätze, Ehegattenerbrecht und Pflichtteilsrecht
}

\author{
Assoz. Prof. Dr. Gregor Christandl LL.M. en Mag. Maximilian Dallago*
}

\section{Allgemeine Grundsätze}

\subsection{Verfassungsrechtliche Garantie und Quellen} Das italienische Erbrecht findet seine verfassungsrechtliche Verankerung in Artikel 42 der Verfassung (Costituzione della Repubblica Italiana). Darin werden die gesetzliche und die testamentarische Erbfolge als gegeben vorausgesetzt und unter Gesetzesvorbehalt gestellt. Sie sind somit vom Gesetzgeber in ihrem Wesensgehalt jedenfalls $\mathrm{zu}$ wahren und bedürfen lediglich der inhaltlichen Ausgestaltung durch einfaches Gesetz. ${ }^{1} \mathrm{Ob}$ das in der Verfassung unerwähnt gebliebene Pflichtteilsrecht als Mindestteilhaberecht der engsten Familienangehörigen am Vermögen des Erblassers ${ }^{2}$ ebenso verfassungsrechtlichen Bestandsschutz genießt, ist in Italien bis heute ungeklärt. ${ }^{3}$

* Assoz. Prof. Dr. Gregor Christandl, LL.M. (Yale) ist Leiter des Instituts für Italienisches Recht der Universität Innsbruck in Österreich (Abschnitte 1 und 2). Universitätsassistent Mag. Maximilian Dallago ist wissenschaftlicher Mitarbeiter am Institut für Italienisches Recht der Universität Innsbruck in Österreich (Abschnitt 3).

1. Zum Erbrecht in der Verfassung vgl. D'Aloia, La successione mortis causa nella costituzione in Bonilni ( $\mathrm{Hg}$.), Trattato di diritto delle successioni e donazioni vol. I (2009) 43 ff.; Sesta, Codice delle successioni e donazioni - Bd. I (2011) $3 \mathrm{ff}$.

2. Bilotti, Profili comparatistici della successione nell'impresa, Studi giuridici europei 2013, 355 mit Bezug auf die Diskussion in Deutschland und die Entscheidung des BVerfG, wonach das GG eine Mindestbeteiligung der Kinder am Nachlass des Erblassers garantiert (BVerfGE 112, 332).

3. Gegen eine verfassungsrechtliche Garantie in der Rsp: Cass civ 24.6.1996, Nr. 5832, Nuova giurisprudenza civile commentata 1997 I, 164; in der Lehre: Gazzoni, Competitività e dannosità della successione
Das italienische Erbrecht ist in erster Linie in Buch II (Art. 456-768-octies) des italienischen Zivilgesetzbuchs (Codice civile, im Folgenden kurz: CC) ${ }^{4}$ geregelt. Darüber hinaus befinden sich erbrechtlich relevante Bestimmungen im Arbeits- und Sozialrecht (Art. 2122 CC), ${ }^{5}$ im Gesellschaftsrecht (Art. 2284, 2469 CC) sowie im Zusammenhang mit Publizitätsvorschriften bei Immobilien und eingetragenen beweglichen Sachen (Art. 2645-ter, 2648, 2652, 2660, 2661, 2662, 2685, 2690 CC). Unter den vielen Sondergesetzen, die erbrechtliche Probleme aufgreifen, sind etwa ${ }^{6}$ das Scheidungsrecht, ${ }^{7}$ Mietrecht ${ }^{8}$ und das Agrarrecht ${ }^{9}$ zu nennen. Ein Sonder-

necessaria (a proposito dei novellati artt. 561 e 563 c.c.), Giustizia civile 2006, II, 4 f.; aA Cinque, Sulle sorti della successione necessaria, Rivista di diritto civile 2011 II, 496; Delle Monache, Abolizione della successione necessaria?, Rivista del notariato 2007, 818; Bilotti, Profili comparatistici (FN 68) 355.

4. Eine deutschsprachige Übersetzung des italienischen Zivilgesetzbuchs von Bauer/Eccher/König/Kreuzer/Zanon ist unter folgender Adresse kostenlos abrufbar: www.provinz.bz.it/anwaltschaft/themen/ zivilgesetzbuch.asp; Gesetzeszitate unter Anführungszeichen sind dieser Übersetzung entnommen.

5. Hierbei handelt es sich um eine gesetzliche Sondererbfolge hinsichtlich der Abfertigung und der Entschädigung wegen Beendigung des Arbeitsverhältnisses wegen Todes. Vgl. dazu Eccher in Eccher/Schurr/Christandl, Handbuch Italienisches Zivilrecht (2009) Rz 6/44.

6. Eine vollständige Aufzählung aller erbrechtlich relevanten Bestimmungen ist hier nicht möglich. Vgl. für einen Überblick Sesta, Codice delle successioni e donazioni - Bd. II (2011).

7. Art. 9-bis L 1.12.9170 Nr. 898: Recht des geschiedenen Ehepartners auf nachehelichen Scheidungsunterhalt zulasten des Nachlasses.

8. Zugunsten bestimmter mit dem Erblasser zusammenlebender naher Verwandter ist ein Eintrittsrecht in den Mietvertrag vorgesehen: Art. 6 Abs. 1 L 27.7.1978 Nr. 392. Dazu Eccher (FN 71) Rz 6/40.

9. Die Bestimmungen sichern im Wesentlichen das Recht auf Fortsetzung der landwirtschaftlichen Tätigkeit auf den landwirtschaftlichen Flächen, so etwa durch ein gesetzliches Pachtverhältnis zugunsten des in der Landwirtschaft tätigen Miterben oder durch ein Eintrittsrecht in beste- 
erbrecht für landwirtschaftliche Betriebseinheiten (sogenannter 'geschlossener Hof', maso chiuso) gibt es aus historischen Gründen ausschließlich in der Provinz Bozen-Südtirol. ${ }^{10}$

\subsection{Eröffnung und Gegenstand der Erbfolge}

Mit dem $\operatorname{Tod}^{11}$ der natürlichen Person wird von Gesetzwegen am Ort des letzten Wohnsitzes des Erblassers die Erbfolge eröffnet (apertura della successione, Art. 456 CC). Vererblich sind grundsätzlich alle wirtschaftlich bewertbaren Rechtspositionen (Forderungen und Schulden einschließlich Vertragspositionen, sofern diese nicht auf die Person des Erblassers abstellen, Sachenund Gestaltungsrechte, Erbrecht ${ }^{12}$ ), unter Ausschluss jener, die mit der Person des Erblassers so eng verbunden sind, dass sie mit seinem Tod untergehen. ${ }^{13}$ Dies gilt insbesondere für Persönlichkeitsrechte, nicht aber für Ansprüche aus deren wirtschaftlicher Verwertung. Vererblich ist nach italienischem Recht auch der Besitz, der dem Erben mit Erbantritt rückwirkend bis zur Eröffnung der Erbfolge zusteht und der jenem des Erblassers hinzuzurechnen ist (Art. 1146 CC). Hinsichtlich bestimmter Vermögensgegenstände tritt eine gesetzliche Sondererbfolge ein, sodass diese nicht in den Nachlass fallen. Dies gilt insbesondere für die Abfertigung nach Beendigung der Arbeitstätigkeit, für Ansprüche aus dem Pensionsfonds bei Versterben vor Fälligkeit des Genussrechts ohne Nennung eines Begünstigten ${ }^{14}$ sowie für den Eintritt in den Miet- bzw. Pachtvertrag. ${ }^{15}$ Ansprüche aus einer Lebensversicherung zugunsten Dritter fallen ebenso nicht in den Nachlass. Sie werden dem Begünstigten unmittelbar aus dem Vertrag auf direktem Weg zugewandt (Art. 1920 Abs. 3 CC). ${ }^{16}$

\subsection{Berufungsgrüinde}

Als erbrechtliche Berufungsgründe kennt das italienische Recht lediglich die gesetzliche und die testamentarische Erbfolge (Art. 457 Abs. 1 CC), wobei diese ohne weiteres nebeneinander zur Anwendung gelangen können (Art. 457 Abs. 2 CC). Dies ist zum Beispiel dann der Fall, wenn der Erblasser nicht über den gesamten Nachlass verfügt hat oder das Testament nur teilweise wirksam ist. Im Übrigen gilt in Italien, im Gegensatz zu den Rechtsordnungen des germanischen Rechtskreises,

hende Pachtverträge. Art. 49 L 3.5.1982 Nr. 203. Dazu Eccher (FN 71) Rz 6/41.

10. Legge della Provincia Autonoma di Bolzano 28.11.2001, Nr. 17 ('Höfegesetz').

11. Bzw. der Todeserklärung/-vermutung, dazu im Detail Christandl in Eccher/Schurr/Christandl, Handbuch Italienisches Zivilrecht (2010) Rz $2 / 17 \mathrm{ff}$.

12. Hat der Verstorbene eine ihm zugefallene Erbschaft noch nicht angenommen, so geht das Annahmerecht auf seine Erben über, sofern diese die Erbschaft ihres Vorfahren annehmen. Bei mehreren Erben können auch nur einzelne die Erbschaft ihres Vorfahren annehmen, sodass sich die Transmission des Erbrechts auf sie beschränkt (Art. 479 CC).

13. Vgl. im Detail Eccher (FN 5) Rz 6/4 ff

14. Dazu Christandl, Vertragliche Sondererbfolge: der Pensionsfonds im italienischen Recht in FS Eccher (2017) $241 \mathrm{ff}$

15. Vgl. zu den gesetzlichen Sondererbfolgen Eccher (FN 5) Rz 6/39 ff.

16. Zu den vertraglichen Sondererbfolgen im Detail vgl. Christandl, Willsubstitutes in Italy in Braun/Röthel $(\mathrm{Hg})$, Passing wealth on death Will-substitutes in comparative perspective (2016) $131 \mathrm{ff}$ ein strenges Erbvertragsverbot (Art. 458 CC). ${ }^{17}$ Als Erbverträge im Sinne dieses Verbots gelten alle Vereinbarungen zur Erbeinsetzung oder zur Anordnung von Vermächtnissen (patti successori istitutivi), Erbverzichtsverträge (patti successori rinunciativi) sowie Verträge, mit welchen über eine erhoffte Erbschaft verfügt wird (patti successori dispositivi). Dieses umfassende Erbvertragsverbot wird darüber hinaus durch das ausdrückliche Verbot des Pflichtteilsverzichts (Art. 557 Abs. 2 CC), das Verbot des gemeinschaftlichen Testaments (Art. 589 CC) und das Verbot des Widerrufsverzichts beim Testament (Art. 679 CC) verstärkt. Daraus ergibt sich ein sehr starres Korsett, das der Privatautonomie des Erblassers enge Grenzen setzt. Dies wirkt sich auch auf die Zulässigkeit von lebzeitigen Verfügungen von Todes wegen aus. Wegen Verstoßes gegen das Erbvertragsverbot ist daher jede Form der Schenkung auf den Todesfall unzulässig, wenn sie auf die Übertragung von zum Todeszeitpunkt des Schenkers in seinem Vermögen verbleibenden Vermögensgegenständen gerichtet ist. ${ }^{18}$

Im Jahr $2006^{19}$ wurde das Erbvertragsverbot insofern etwas gelockert, als seither mit dem sogenannten Familienvertrag (patto di famiglia) in Bezug auf die Unternehmensübergabe oder Übergabe von Gesellschaftsanteilen zu Lebzeiten eine Ausnahme geschaffen wurde. Diese erlaubt nunmehr erstmals einen vertraglichen Pflichtteilsverzicht der weichenden Erben im Interesse des Fortbestands des Familienunternehmens in der Generationenfolge. $^{20}$ Es handelt sich dabei um einen mit Notariatsakt zu errichtenden lebzeitigen Vertrag mit unmittelbarer dinglicher Wirkung, mittels dessen der Unternehmer seinen Kindern oder einem seiner Kinder sein Unternehmen bzw. seine Gesellschaftsanteile übergibt. An dieser Übergabe müssen alle zu diesem Zeitpunkt der Übergabe abstrakt Pflichtteilsberechtigten beteiligt sein (Art. 768-quater Abs. 1 CC). Sie erhalten vom Übernehmer eine am Übertragungswert orientierte Abfindung, können darauf aber auch ganz oder teilweise verzichten (Art. 768-quater Abs. 2 CC). Der damit einhergehende Pflichtteilsverzicht der weichenden Erben bezieht sich ausschließlich auf das übertragene Unternehmen bzw. die Gesellschaftsanteile und lässt somit die Pflichtteilsansprüche hinsichtlich des restlichen Vermögens des Übergebers unberührt. Aufgrund der rechtlichen Ausgestaltung des Familienvertrags hat er

17. 'Art 458 Verbot der Erbverträge - Vorbehaltlich der Bestimmungen in Art 768-bis ff, ist jede Vereinbarung, mit der jemand über seine Erbfolge verfügt, nichtig. Ebenso nichtig ist jede Handlung, mit der jemand über die Rechte verfügt, die ihm aus einer noch nicht eröffneten Erbschaft zustehen können oder mit der er auf diese verzichtet.' Zum Erbvertragsverbot vgl. Eccher (FN 71) Rz 6/21 ff.; Bonilini, Manuale di diritto ereditario e delle donazioni (2016) $22 \mathrm{ff}$.

18. Dazu näher Eccher (FN 5) Rz 6/46.

19. L 14.2.2006, Nr. 55.

20. Dazu Kindler, Neue Gestaltungsmöglichkeiten im italienischen Unternehmenserbrecht: der Familienvertrag, FamRZ 2007, 952 ff.; Braun, Towards a greater autonomy for testators and heirs, ZEuP 2012, $469 \mathrm{ff}$. Zur Behandlung des Familienvertrags im IPR: Laimer, Vertrag mit erbrechtlichen Folgen? in FS Eccher 2017, 623 ff. 
sich trotz steuerlicher Vorteile ${ }^{21}$ bisher als für die Praxis wenig attraktiv erwiesen, ${ }^{22}$ weshalb die Regelung von vielen als verunglückt angesehen wird. ${ }^{23}$

\section{Gesetzliches Erbrecht des Ehegatten}

2.1 Grundstruktur der gesetzlichen Erbfolge

Das gesetzliche Erbrecht folgt in seiner Struktur einem an der Verwandtschaft anknüpfenden, nach Linien und Graden gemischtem System (sistema lineare graduale). Die Verwandten gliedern sich dabei in folgende drei Gruppen (categorie di successibili), die teils nacheinander teils nebeneinander zur Erbfolge berufen werden (Art. 565 CC): (a) der Ehegatte bzw. der ihm gleichgestellte eingetragene Partner, (b) die Kinder und deren Nachkommen, (c) die Vorfahren sowie (d) die Seitenverwandten bis zum sechsten Grad, wobei gradnähere Verwandte gradfernere Verwandte stets ausschließen. Sind keine Angehörige dieser Gruppen zum Zeitpunkt der Eröffnung der Erbfolge vorhanden, fällt der Nachlass an den Staat. Dieser übernimmt das Nachlassvermögen, wobei die Haftung für Nachlassschulden auf den Nachlasswert beschränkt bleibt (Art. 586 CC).

Gemeinsam mit den Eltern sind die Geschwister als Mitglieder der Kernfamilie des kinderlosen Erblassers mit einer eigenen Erbberechtigung zur Erbfolge berufen. Auch die Gegenseitigkeit der Erbberechtigung bleibt im italienischen gesetzlichen Erbrecht stets gewahrt. Im Vergleich zum Parentelsystem österreichisch-deutscher Prägung werden diese Vorteile um den Preis einer wesentlich komplexeren Erbrechtsstruktur erkauft.

21. Die Unternehmensübergabe bleibt steuerfrei, wenn der Übernehmer für mindestens fünf Jahre das Unternehmen weiterführt. Vgl. dazu Memento Pratico Famiglia e Patrimonio (2014) Rz 2098.

22. Die Gründe für diesen Misserfolg sind vielfältig. Insbesondere erweisen sich aber die zwingende Teilnahme aller abstrakt Pflichtteilsberechtigten sowie die Instabilität des Familienvertrags aufgrund späterer Anfechtungsmöglichkeit als Hindernisse. Auch die Vorgabe, dass die Abfindungen grundsätzlich vom Übernehmer zu leisten sind, erweist sich für die Praxistauglichkeit als hinderlich. Zuletzt zum Misserfolg und zu Möglichkeiten der Revitalisierung des Rechtsinstituts Laurini, Insuccesso dell'istituto e prospettive di rivitalizzazione nella prassi negoziale in laccarino $(\mathrm{Hg})$, Successioni e donazioni - Bd. II (2017) 1994 ff.

23. Bilotti, Profili comparatistici (FN 68) 307; Sardu, La trasmissione ereditaria dell'impresa di famiglia in Falzone Calvisi $(\mathrm{Hg})$, Diritto successorio. Approfondimenti tematici (2012) 218; De Rosa/Russo/Lo Presti, I patti di famiglia in Oriani/Zanaboni $(\mathrm{Hg})$, Governance del patrimonio e passaggio generazionale (2013) 126; Bonilini, Manuale (FN 17) 207; Irrera, Passaggio generazionale dell'impresa e strumenti giuridici di supporto: un percorso complesso, II nuovo diritto delle società, Heft 15, 2014, 16; Bucelli, Diritto ereditario e destinazione produttiva dei beni (un cenno comparativo al sistema francese), Contratto e impresa 2015, 762

\subsection{Der Ehegatte/eingetragene Partner}

\subsubsection{Stärkung der Position des Ehegatten und eheliches Güterrecht}

In der Aufzählung der nach gesetzlichem Erbrecht Erbberechtigten in Artikel 565 CC steht der Ehegatte an erster Stelle. Dies hat zum einen große symbolische Bedeutung, denn vor der Familienrechtsreform des Jahres $1975^{24}$ war der Ehegatte noch an vorletzter Stelle genannt worden; zum anderen wird darin aber auch die besonders starke Position des Ehegatten im gesetzlichen Erbrecht sichtbar. Dies war indes nicht immer so. Noch in der Fassung des Codice civile im Jahr seines Inkrafttretens 1942 stand dem Ehegatten lediglich ein Nießbrauchrecht an einem bestimmten Teil des Nachlasses in Konkurrenz mit ehelichen Kindern zu. Nur in Ermangelung von ehelichen Kindern konnte der Ehegatte auch Erbe werden, konkurrierte dabei aber mit Verwandten bis zum vierten Grad. Erst wenn es auch an Verwandten bis zum vierten Grad fehlte, wurde der überlebende Ehegatte zum Alleinerben. ${ }^{25}$

Mit der Familienrechtsreform des Jahres 1975 wurde die Position des Ehegatten wesentlich gestärkt. Einerseits wurde dem Ehegatten nun jedenfalls, d.h. auch in Konkurrenz mit ehelichen Kindern, ein Erbrecht eingeräumt. Andererseits wurde mit der Einführung eines neuen gesetzlichen Güterstands im Eherecht (sogenannte gesetzliche Gütergemeinschaft, comunione legale) erreicht, dass stets nur jener Teil des während der Ehe aufgebauten Vermögens in den Nachlass fällt, der nach der güterrechtlichen Auseinandersetzung im Vermögen des verstorbenen Ehegatten verbleibt. Alle während der Ehe erworbenen Anschaffungen fallen, bis auf wenige Ausnahmen, in die Gütergemeinschaft ${ }^{26}$ und sind somit bei Tod eines Ehepartners zu gleichen Teilen aufzuteilen, sodass neben den persönlichen Gütern des Erblassers nur über den ihm zugewiesenen Quotenanteil an den Gemeinschaftsgütern die Erbfolge eröffnet wird.

\subsubsection{Gleichgeschlechtliche Partner einer unione civile}

Mit der unione civile besteht seit 2016 auch für gleichgeschlechtliche Paare die Möglichkeit, eine formalisierte Partnerschaft einzugehen. ${ }^{27}$ Die eingetragenen Partner sind Ehepartnern im gesetzlichen Erbrecht und im Pflichtteilsrecht vollkommen gleichgestellt (Art. 1 Abs. $21 \mathrm{~L} \mathrm{Nr.} \mathrm{76/2016).}{ }^{28}$

\subsubsection{Erbrecht neben Kindern, Vorfahren und Geschmistern}

Der Ehegatte und der diesem seit 2016 gleichgestellte Partner einer eingetragenen Partnerschaft (unione civile)

24. Legge 19.5.1975, Nr. 151 sulla riforma del diritto di famiglia.

25. Braun, Intestate succession in Italy in Reid/De Waal/Zimmermann $(\mathrm{Hg})$, Intestate succession (2015) $83 \mathrm{f}$.

26. Vgl. Art. $177 \mathrm{CC}$; ausführlich zum gesetzlichen Güterstand in Italien: Christandl (FN 77) Rz 5/111 ff

27. Die eingetragene Partnerschaft (unione civile) für gleichgeschlechtliche Paare wurde mit L 20.5.2016 Nr. 76 in Italien eingeführt.

28. Vgl. dazu Venuti, I diritti successori della persona unita civilmente e del convivente di fatto: un confronto con il sistema tedesco, Europa e diritto privato $2017,1241 \mathrm{ff}$. 
konkurriert ausschließlich mit den Kindern und Nachfahren des Erblassers bzw. mit dessen Vorfahren und Geschwistern und deren Nachkommen kraft Eintrittsrecht. Sonstige Seitenverwandte kommen in Konkurrenz mit dem hinterbliebenen Ehegatten/Partner nicht zum Zug, sodass dieser Alleinerbe wird (Art. 583 CC). Das Erbrecht des Ehegatten/Partners erlischt mit Scheidung, ${ }^{29}$ nicht aber bei Ehegatten mit der gerichtlichen Trennung (separazione legale dei coniugi), es sei denn, diese wurde aufgrund Verschuldens des Hinterbliebenen ausgesprochen (Art. 585 CC).

Konkurriert der Ehegatte/Partner mit den Kindern des Erblassers ist zu unterscheiden, ob dieser ein oder mehrere Kinder hinterlässt. Dem Ehegatten steht neben einem Kind die Hälfte des Nachlasses, neben mehreren Kindern ein Drittel des Nachlasses zu (Art. 581 CC).

Verstirbt der Erblasser ohne Kinder zu hinterlassen, so konkurriert der Ehegatte/Partner mit den Vorfahren und den Geschwistern des Erblassers und erhält neben diesen zwei Drittel des Nachlassvermögens (Art. 582 $\mathrm{CC})$.

$\mathrm{Zu}$ diesen Quoten kommt jedenfalls ein dingliches Wohnrecht an der im (Mit-)Eigentum des Erblassers stehenden Familienwohnung sowie ein dingliches Gebrauchsrecht an den in dieser Wohnung befindlichen, im (Mit-)Eigentum des Erblassers stehenden beweglichen Sachen ${ }^{30}$ hinzu (Art. 540 Abs. 2 CC ana$\log ) .31$

\subsubsection{Kein Erbrecht für Lebensgefährten}

Das Gesetz Nr. 76/2016, mit dem die eingetragene Partnerschaft für gleichgeschlechtliche Paare eingeführt wurde, sieht auch eine rechtliche Regelung von nichtehelichen Lebensgemeinschaften vor (Art. 1 Abs. 36-65 L Nr. 76/2016). Diese stellt den Lebensgefährten nicht mit dem Ehegatten/Partner gleich und führt ihn auch nicht als gesetzlichen Erben im letzten Rang ein. Dennoch wird sein Eintrittsrecht in den Mietvertrag des verstorbenen Lebensgefährten garantiert (Art. 1 Abs. 44 L Nr. 76/2016). Im Übrigen steht ihm an der im Eigentum des Verstorbenen stehenden Wohnung ein Wohnrecht für zwei Jahre zu, solange er in dieser Wohnung lebt und keine andere Lebensgemeinschaft, Ehe oder Partnerschaft eingegangen ist. Die Dauer des Wohn-

29. Der Verlust des Erbrechts tritt dann ein, sobald das Scheidungsurteil in Rechtskraft erwachsen ist. Bonilini, Manuale (FN 17) 165. Wenn der Ehegatte nachehelichen Unterhalt in Form regelmäßig wiederkehrender Leistungen erhalten hat, kann er zulasten des Nachlasses weiterhin Unterhalt beziehen. Vgl. dazu Art. 9bis L 898/1970 (Scheidungsgesetz). Dazu auch Bonilini, Manuale (FN 17) $166 \mathrm{f}$.

30. Der Begriff der zum Haushalt gehörenden beweglichen Sachen deckt sich zwar in weiten Teilen, jedoch nicht vollständig mit jenem des österreichischen Rechts ( $\$ 745$ Abs. 1 ABGB). Nach italienischem Recht ist jedenfalls wesentlich, dass die beweglichen Sachen dauerhaft Wohnzwecken dienen. Bonilini, Manuale (FN 17) 162.

31. Diese nur im Pflichtteilsrecht vorgesehenen Rechte kommen nach inzwischen gefestigter Rechtsprechung zum gesetzlichen Erbteil des Ehegatten/Partners hinzu (iS gesetzlicher Vorausvermächtnisse) und sind nicht als Teil desselben zu berechnen. Cass sez un 27.2.2013, Nr. 4847, Giustizia civile 2013, $1756 \mathrm{ff}$ rechts erhöht sich entsprechend der Dauer der Lebensgemeinschaft auf bis zu fünf Jahre. Sind auch Kinder des Verstorbenen im Haushalt wohnhaft, beträgt die Mindestdauer drei Jahre (Art. 1 Abs. 42 L Nr. 76/2016).

\section{Pflichtteilsrecht}

\subsection{Grundsätze}

Die Familie nimmt in der italienischen Gesellschaft und damit auch im italienischen Recht eine zentrale Rolle ein. ${ }^{32}$ Die Normen zum Pflichtteilsrecht ${ }^{33}$ haben deshalb zum Schutz des Familienvermögens ${ }^{34}$ grundsätzlich zwingenden Charakter: Artikel 457 Absatz 3 CC hält diesbezüglich einleitend fest, dass die testamentarischen Verfügungen nicht die den Pflichtteilsberechtigen gesetzlich vorbehaltenen Ansprüche beeinträchtigen dürfen. Offenkundig ist demnach die Einschränkung der Privatautonomie ${ }^{35}$ des Erblassers durch das Pflichtteilsrecht. ${ }^{36}$ Allerdings handelt es sich hierbei nicht um ein Testierverbot ${ }^{37}$ oder um eine Beschränkung der Verfügungsfreiheit zum Zeitpunkt des Testierens, die Nichtigkeit oder Unwirksamkeit hervorrufen könnte; die Einschränkung folgt vielmehr erst aus der erfolgreichen Kürzungsklage des Pflichtteilsberechtigten. ${ }^{38}$ Verboten ist außerdem der Pflichtteilsverzicht zu Lebzeiten des Erblassers (Art. 557 Abs. 2), während der Verzicht auf den Kürzungsanspruch nach dem Tod des Erblassers erlaubt ist. ${ }^{39}$

Das italienische Erbrecht unterscheidet bei Vorhandensein von Pflichtteilsberechtigten zwischen verfügbarem Teil (quota disponibile) des Vermögens, über den der Verstorbene frei verfügen kann und nichtverfügbarem Teil (quota indisponibile/legittima), der den Pflichtteilsberechtigten vorbehalten ist. Gerade deshalb spricht

32. Auf verfassungsrechtlicher Ebene sind der Schutz und die Solidarität in der Familie in den Art. 2 und 29 Verf verankert; Moncalvo, Sulla natura giuridica dell'azione di riduzione, Familia 2004, 180; Visconti, Legittimari in laccarino $(\mathrm{Hg})$, Successioni e donazioni (2017) $1303 \mathrm{f}$.

33. Eine ausführliche Analyse der Entwicklung des Pflichtteilsrechtes liefern De Belvis, La successione necessaria tra storia e riforme (2013) $3 \mathrm{ff}$.; Mengoni in Cicu/Messineo, Successioni per causa di morte, Parte speciale, Successione necessaria, XLIII/2 (2000) $1 \mathrm{ff}$.

34. Ferri in Scialoja/Branca, Art. 536-564 (1981) 2; Bianca, Diritto civile 2.2. successioni (2015) 175.

35. Diese Beschränkung stützt sich auf Art. 42 letzter Absatz der italienischen Verfassung, der die Ausgestaltung der Grenzen der Erbfolge unter Gesetzesvorbehalt stellt. Cass civ 24.6.1996, Nr. 5832, Rivista del Notariato 1997, 935; siehe dazu auch Bianca (Fn 34) 175.

36. Kritisch dazu Delle Monache, Scenari attuali in materia di tutela del legittimario, Nuova giurisprudenza civile commentata 2008, II, 59, 68.

37. Gemäß Art. 549 CC kommt es nur dann zur Nichtigkeit, wenn der Pflichtteil mit Lasten und Bedingungen belegt wird; siehe 3.3.

38. Mengoni (Fn 33) 64; Franco, Come si tutela la legittima: le tre azioni in laccarino (Hg), Successioni e donazioni (2017) 1388. In der Rsp: Cass civ 12.4.2002, Nr. 5323, Giustizia civile massimario 2002, 638; Cass civ 11.6.2003, Nr. 9424, Giustizia civile massimario 2003, 1421.

39. Die Pflichtteile der anderen Pflichtteilsberechtigten bleiben aber davon unberührt; siehe Cass sez un 12.6.2006, Nr. 13524, Giurisprudenza italiana 2007, 1116; eine Ausnahme stellt der schon angesprochene Familienvertrag dar, siehe 1.3. 
man in Bezug auf den Pflichtteil auch nach französischem Vorbild von Reserve/riserva.

Das Pflichtteilsrecht, welchem der italienische Gesetzgeber den 10. Abschnitt des zweiten Buches des Codice civile (Art. 536 ff. CC) widmet, ist somit als echtes Noterbrecht ausgestaltet (successione necessaria). Das bedeutet, dass die Pflichtteilsberechtigten nicht zwangsläufig Erben sein müssen, jedenfalls aber ihnen 'das Gesetz einen Anteil der Erbschaft oder andere Erbfolgerechte vorbehält' (Art. 536 CC) und diese demnach Anspruch auf reale Teilhabe am Vermögen des Erblassers haben. ${ }^{40}$

\subsection{Die Pflichtteilsberechtigten}

Pflichtteilsberechtigt sind laut Artikel 536 CC der Ehegatte, dem seit 2016 der eingetragene Partner ${ }^{41}$ gleichgestellt ist, die Kinder ${ }^{42}$ und bei Nichtvorhandensein von Kindern konkurrieren mit dem Ehegatten/eingetragenen Partner die Vorfahren. Außerdem werden auch die Nachkommen der Kinder zu Pflichtteilsberechtigten (Art. 536 Abs. 3 CC), wenn diese im Falle von Repräsentation durch Eintrittsrecht in deren Pflichtteil eintreten, ${ }^{43}$ wobei eine allenfalls angeordnete Ersatzerbschaft (sostituzione) dem nicht entgegensteht. ${ }^{44}$

Das Gesetz sieht je nach Konstellation der konkurrierenden Pflichtteilsberechtigten eigenständige Pflichtteilsquoten vor: ${ }^{45}$ Hinterlässt der Verstorbene nur Kinder bzw. deren Nachkommen, so gebührt dem einzigen Kind die Hälfte des Vermögens, während bei mehreren Kindern diesen zwei Drittel des Vermögens zustehen (Art. 537 CC). Das bedeutet, dass in diesem Falle der Verstorbene über die Hälfte bzw. ein Drittel seines Vermögens frei verfügen konnte, während die andere Hälfte bzw. zwei Drittel als nichtverfügbar einzustufen und somit den Pflichtteilsberechtigten vorbestimmt sind.

Hinterlässt der Verstorbene mit dem Ehegatten/eingetragenen Partner ein einziges Kind, so beträgt der Pflichtteil je ein Drittel; bei mehreren Kindern gebührt den Kindern die Hälfte, dem Ehegatten/eingetragenen Partner ein Viertel des Vermögens (Art. 542 CC).

Hinterlässt der Verstorbene nur den Ehegatten/eingetragenen Partner, so ist diesem die Hälfte des Vermögens vorbehalten, sowie jedenfalls, wie schon angesprochen, das Wohnrecht an der zum Familienwohnsitz bestimmten Wohnung ${ }^{46}$ und das Gebrauchsrecht an den Haushaltsgegenständen (Art. 540 CC). Die herrschende Lehre erkennt darin ein gesetzliches Vermächtnis, das

Christandl, Der italienisch-österreichische Erbfall, Grundzüge des italienischen Erbrechts für die notarielle Praxis, Notariatszeitung 2017, 211; Delle Monache, Scenari attuali (Fn 36) 62

41. Eingeführt durch die L 20.5.2016 Nr. 76 .

42. 'Den Kindern gleichgestellt sind adoptierte Kinder' (Art. 536 Abs. 2 CC).

43. Magliulo, Intangibilità della legittima in laccarino $(\mathrm{Hg})$, Successioni e donazioni (2017) $1368 \mathrm{ff}$.

44. Siehe Ferri (Fn 34) $26 \mathrm{f}$.

45. Visconti (Fn 32) $1306 \mathrm{ff}$.

46. Cass civ 12.6.2014, Nr. 13407, Foro italiano 2014, I, 2060. unter die von Artikel 536 CC genannten anderen Rechte an der Erbschaft fällt. ${ }^{47}$

Während dem geschiedenen Ehepartner/eingetragenen Partner kein Erbrecht zusteht, ist beim getrennten Ehegatten zu unterscheiden: Wurde diesem die Trennung aufgrund Verschuldens rechtskräftig angelastet, hat dieser Anspruch auf lebenslange Unterhaltsleistungen im Rahmen des eingeschränkten Unterhalts, den er zum Zeitpunkt der Eröffnung der Erfolge bezog (Art. 548 CC). Wurde dem Ehegatten die Trennung nicht angelastet, so behält er den Pflichtteilsanspruch eines Ehegatten. Bei der eingetragenen Partnerschaft gibt es nur die Scheidung ohne vorherige Trennung, ${ }^{48}$ sodass diese Sonderbestimmungen nur für Ehegatten gelten.

Vorfahren zählen nur subsidiär zu den Pflichtteilsberechtigten und kommen somit nur dann zum Zug, wenn der Verstorbene keine Kinder hinterlässt. Konkurrieren die Vorfahren mit dem Ehegatten/eingetragenen Partner, so steht ihnen ein Viertel des Vermögens zu, während dem Ehegatten/eingetragenen Partner die Hälfte vorbehalten ist (Art. 544 CC). Hinterlässt der Verstorbene nur Vorfahren, so ist diesen ein Drittel vorbehalten (Art. 538 CC). Gradnähere Vorfahren schließen dabei, der allgemeinen Regel der gesetzlichen Erbfolge folgend, gradfernere aus (Art. 569 CC).

\subsection{Berechnungsgrundlage und Zuwendung des Pflichtteils}

Der Pflichtteilsberechtigte hat Recht auf reale Teilhabe am Vermögen und gerade deshalb verbietet der Gesetzgeber das Auferlegen von Lasten oder Bedingungen (Art. 549 CC). Diese sogenannte 'Unantastbarkeit des Pflichtteils' (intangibilità della legittima) ist nunmehr ${ }^{49}$ allerdings in quantitativer und nicht in qualitativer Weise zu verstehen; ${ }^{50}$ das bedeutet, dass der Wert der zugeteilten Güter dem Wert der jeweiligen Quoten entsprechen muss. ${ }^{51}$ Ausschlaggebend ist demnach welcher Wert den genannten Quoten entspricht: Konkrete Berechnungsgrundlage für den Pflichtteil stellt durch die sogenannte 'riunione fittizia' das Vermögen des Verstorbenen, und nicht der Nachlass selbst dar. ${ }^{52}$ Durch diese 'fiktive Berechnung' soll festgestellt werden, ob es eine Pflichtteilsverletzung gegeben hat. Zuerst werden

47. Cattaneo, La vocazione necessaria e la vocazione legittima in Rescigno $(\mathrm{Hg})$ Trattato di diritto privato, 5 (1997) 437 f., 445 ff.; Mengoni (Fn 33) 167

48. Siehe Abs. $22 \mathrm{ff}$. der L 76/2016. In der Lehre: Al Mureden, Lo scioglimento dell'unione civile tra rapporto di coppia e ruolo del 'genitore sociale', Nuova giurisprudenza civile commentata 2016, 1699 ff.; Savi, Lo scioglimento 'volontario' dell'unione civile, Rivista trimestrale di diritto e procedura civile 2017, $681 \mathrm{ff}$.; Longo, I nuovi modelli di famiglia. Unione civile, convivenza, famiglia di fatto (2017) $78 \mathrm{ff}$.

49. Romano, Lo sviluppo dell'azione di riduzione nel conflitto tra tutela dei legittimari e certezza dei traffici giuridici, Studium luris 2014, $686 \mathrm{ff}$.

50. Bonilini, Manuale (FN 17) 97; Mengoni (Fn 33) 89 ff.; Capozzi, Successioni e donazioni, Bd. I (2015) 467 f.; in der Rsp: Cass civ 12.9.1970, Nr. 1403, Foro italiano, 1970, I, 2399; Cass civ 9.2.2005, Nr. 2617, unveröffentlicht, abrufbar unter iusexplorer.it; Cass civ 11.8.2015, Nr. 16698, unveröffentlicht, abrufbar unter iusexplorer.it.

51. Eccher (FN 71) Rz 6/172.

52. Christandl, Der italienisch-österreichische Erbfall (Fn 40) 211. 
vom Nachlass (relictum) die Schulden abgezogen; diesem bereinigten Nachlass werden fiktiv die Schenkungen (donatum) hinzugerechnet. ${ }^{53}$ Zum Donatum zählen sowohl direkte (Art. 769 ff. CC) als auch mittelbare Schenkungen (Art. 809 CC) nach ihrem Wert zum Zeitpunkt der Eröffnung der Erbfolge; Verschlechterungen und Verbesserungen des Schenkungsbegünstigten werden nicht berücksichtigt (Art. 748 CC),${ }^{54}$ da diese den Pflichtteil verfälschen würden.

Des Weiteren ist es für die Feststellung einer möglichen Pflichtteilsverletzung nötig, eventuelle Vermächtnisse oder Schenkungen zu Gunsten des Pflichtteilsberechtigten $\mathrm{zu}$ berücksichtigen (imputazione ex se). Die Schenkungen und Vermächtnisse werden demnach grundsätzlich dem Pflichtteil angerechnet (donazioni $e$ legati in conto di legittima), ${ }^{55}$ es sei denn der Verstorbene hat den Pflichtteilsberechtigten explizit davon befreit (Art. 564 Abs. 2 CC). Zu unterscheiden gilt diese Anrechnung vom Vermächtnis anstelle des Pflichtteils (Art. $551 \mathrm{CC}$ ) (legato in sostituzione di legittima). Hierbei hat der Begünstigte die Wahl, das Vermächtnis zu behalten und somit auf eine Ergänzung auf den Pflichtteil und Eigenschaft eines Erben definitiv zu verzichten, oder aber das Vermächtnis auszuschlagen und den Pflichtteil zu verlangen.

Aus dem Gesagten geht hervor, dass trotz Einschränkung der Privatautonomie dem Erblasser mehrere Gestaltungsmöglichkeiten zur Deckung des Pflichtteils offen bleiben, da es sich lediglich um Vermögenswerte aus seinem Vermögen handeln muss: ${ }^{56}$ Der Erblasser kann auch testamentarisch Erbquoten zuweisen, welche dem Pflichtteil entsprechen, sowie diese Erbeinsetzungen mit Teilungsanordnungen mit Ausgleichspflicht verbinden. ${ }^{57}$ Dem angesprochenen Prinzip der 'Unantastbarkeit des Pflichtteils' (intangibilità della legittima) allerdings folgend, muss der Pflichtteil frei von Lasten und Bedingungen sein (Art. 549 CC), sodass die Pflichtteilsberechtigten frei über ihren Teil verfügen können. ${ }^{58}$

Eine interessante Ausprägung erfährt das letztgenannte Prinzip im Falle von über den verfügbaren Teil hinausgehenden Zuwendungen (Art. 550 CC): Ordnet der Erblasser einen Fruchtgenuss oder eine lebenslange Rente zu Gunsten Dritter durch Schenkung oder Vermächtnis an, deren Ertrag über jenen aus dem verfügbaren Teil hinausgeht, so findet der sogenannte sozinische Vorbehalt (cautela sociniana) ${ }^{59}$ Anwendung. Durch diese Zuwendungen ergeben sich Belastungen, welche in den Pflichtteil fallen und somit die freie Verfügung über

53. Cass civ 1.12.1993, Nr. 11873, Giustizia civile massimario 1993, 1701; Cass civ 24.7.2012, Nr. 12919, Giustizia civile massimario 2012, 954.

54. Bianca (Fn 34) 403; Eccher (FN 71) Rz 6/171; Christandl, Der italienisch-österreichische Erbfall (Fn 40) 211.

55. Eccher (FN 71) Rz 6/176 ff.

56. Christandl, Der italienisch-österreichische Erbfall (Fn 40) 212.

57. Eccher (FN 71) Rz 6/172.

58. Bonilini, Manuale (FN 17) 180

59. Benannt nach dem italienischen Juristen Marianus Socinus dem Jüngeren (1482-1556). Zur Geschichte und Entwicklung des Rechtsinstituts siehe Mengoni (Fn 33) $339 \mathrm{ff.}$ denselben einschränken können. Um die problematische Wertfeststellung der Belastung zu vermeiden, welche von der Lebensdauer des Begünstigten abhängt, ${ }^{60}$ wird durch den Vorbehalt dem Pflichtteilsberechtigten die Wahl gelassen, entweder die über den Pflichtteil hinausgehende Zuwendung mit der Belastung als Ganzes anzunehmen oder nur den befreiten Pflichtteil zu fordern und somit dem Dritten den über den Pflichtteil hinausgehenden Teil zu überlassen. ${ }^{61}$ Der Vermächtnisnehmer oder Schenkungsempfänger, welcher den freigewordenen verfügbaren Teil erlangt und über diesen ein vollständiges Eigentumsecht erwirbt, ${ }^{62}$ nimmt aber damit nicht die Stellung eines Erben ein.

\subsection{Schutz der Pflichtteilsberechtigten}

Der italienische Gesetzgeber sieht eng miteinander verbundene Schutzmechanismen ${ }^{63}$ sowohl für den gänzlich übergangenen Pflichtteilsberechtigten (legittimario pretermesso) als auch für den in seinem Pflichtteil teilweise Verletzten vor. Die Normen aus dem 2. Teil des 10. Abschnittes des 2. Buches des CC ('Wiederherstellung des den Pflichtteilsberechtigten vorbehaltenen Anteils' 553-564 CC) kommen demnach zur Anwendung, wenn aus den bereits genannten Berechnungen eine Verletzung des Pflichtteils hervorgeht.

Die Kürzungsklage hat die Kürzung der über den verfügbaren Teil hinausgehenden testamentarischen Verfügungen und Schenkungen und somit die Feststellung der relativen Unwirksamkeit ${ }^{64}$ dieser Verfügungen zum Ziel. ${ }^{65}$ In der Tat handelt es sich hierbei um eine Feststellungsklage mit rechtsgestaltender Wirkung (azione di accertamento costitutivo ${ }^{66}$ ), da durch die gerichtliche Entscheidung das (Not-)Erbrecht des Klägers begründet oder erweitert wird. ${ }^{67}$ Zuerst werden verhältnismäßig die testamentarischen Verfügungen, ohne Unterescheidung zwischen Erbeinsetzungen und Vermächtnissen gekürzt (Art. 554, 558 CC) ${ }^{68}$ Schenkungen werden hingegen erst subsidiär gekürzt 'wenn der Wert der Sachen, über die im Testament verfügt worden ist, erschöpft ist' (Art. 555 CC). Dabei wird allerdings bei den letzten begonnen und 'der Reihe nach auf die früheren zurückgegangen' (Art. 559 CC). ${ }^{69}$

Einerseits kann sich die Kürzungsklage gegen die direkt von der testamentarischen Verfügung oder Schenkung Begünstigten richten, andererseits kann sie sich aber sogar unter Umständen gegen Dritterwerber richten

60. Cattaneo (Fn 47) 454; Bonilini, Manuale (FN 17) 188

61. Cass civ 18.1.1995, Nr. 511, Nuova giurisprudenza civile commentata 1996, I, 113; Cass civ 12.3.2012, Nr. 3894, Rivista notariato 2012, 936.

62. Cattaneo (Fn 47) 454.

63. Franco (Fn 38) $1386 \mathrm{ff}$.

64. Cass civ 12.4.2002, Nr. 5323 Giustizia civile massimario 2002, 638; Cass civ 30.7.2002, Nr. 11286, Giurisprudenza italiana 2003, 442; Cass civ 11.6.2003, Nr. 9424, Giustizia civile massimario 2003, 1421.

65. Bonilini, Manuale (FN 17) 193

66. Moncalvo (Fn 32) 187.

67. Mengoni (Fn 33) $80 \mathrm{f}$.

68. Auch hier kann der Erblasser testamentarisch festlegen, 'dass eine seiner Verfügungen vor den anderen Gültigkeit haben soll' (Art. 558 CC).

69. Cass civ 10.3.2016, Nr. 4721, unveröffentlicht, abrufbar unter www. dirittoegiustizia.it. 
(Art. 563 CC). Voraussetzungen für die erfolgreiche Kürzungsklage sind laut Artikel 564 CC die schon angesprochene verpflichtende Anrechnung von Schenkungen und Vermächtnissen (imputazione ex se), sowie, um die Kürzung gegenüber Schenkungsempfängern und Vermächtnisnehmern geltend zu machen, die Erbschaftsannahme des teilweise Übergangenen ${ }^{70}$ mit Vorbehalt der Inventarerrichtung. Nicht erforderlich ist hingegen die Annahme mit Vorbehalt der Inventarerrichtung in den Fällen, in welchen sich die Kürzungsklage gegen Miterben richtet oder der Pflichtteilsberechtigte gänzlich übergangen wurde ${ }^{71}$ und somit eine Inventarerrichtung nicht zweckmäßig wäre. ${ }^{72}$

Die herrschende Lehre gewährt des Weiteren auch den Gläubigern des untätigen, aber nicht verzichtenden Pflichtteilsberechtigten die Möglichkeit der Klagerhebung. ${ }^{73}$

Um genau zu sein, muss die Kürzungsklage im engeren Sinne von der Folgeklage auf Herausgabe unterschieden werden: ${ }^{74}$ Wird eine Verletzung des Pflichtteils durch eine Schenkung oder Vermächtnis festgestellt, so ist bei geschenkten Immobilien ein Teil abzutrennen, wenn dies leicht zu bewerkstelligen ist (Art. $560 \mathrm{CC}$ ). Beträgt der pflichtteilswidrige Teil mehr als ein Viertel des verfügbaren Teils und ist die Abtrennung nicht einfach möglich, so ist die ganze Liegenschaft, 'unbeschadet des Rechtes auf Leistung des Wertes des verfügbaren Teiles', herauszugeben (Art. 560 Abs. 2 CC). Wird das Übermaß um weniger als ein Viertel überstiegen, so kann die ganze Liegenschaft behalten werden und ein Ausgleich in Geld an die Pflichtteilsberechtigten ausbezahlt werden. ${ }^{75}$ Des Weiteren wird durch die Herausgabe der Liegenschaft oder der eingetragenen beweglichen Sache diese von jeder Last oder Hypothek befreit. ${ }^{76}$

Das besonders starke und einschränkende Pflichtteilsrecht tritt aber insbesondere durch den Herausgabeanspruch gegen Dritterwerber zum Vorschein: Reichen die Vermögensgegenstände des Beschenkten nicht aus und sind $\mathrm{ab}$ der Eintragung der Schenkung 77 nicht zwanzig Jahre vergangen, ${ }^{78}$ so kann der Pflichtteilsberechtigte von den Dritten die Herausgabe mit eigener Klage fordern (Art. 563 CC). Unbeschadet bleibt der Erwerb des gutgläubigen Dritten durch Besitzerwerb gemäß geeigneten Rechtstitels (Art. 1153 CC). Weiters kann sich der Dritterwerber 'von der Pflicht, die geschenkten Sachen in Natur herauszugeben, durch Bezahlung des Gegenwertes in Geld befreien' (Art. 563 Abs. 3 CC).

Die Kürzungsklage unterliegt der ordentlichen Verjährungsfrist von zehn Jahren (Art. 2946 CC) ab Annahme der Erbschaft, bzw. ab Eröffnung der Erbfolge oder Veröffentlichung des Testaments bei gänzlicher Übergehung. ${ }^{79}$

\section{Ausblick}

Das italienische Erbrecht zeichnet sich durch ein besonders großzügiges Ehegattenerbrecht ('Horizontalisierung'), ein äußerst strenges, als Noterbrecht ausgestaltetes Pflichtteilsrecht und ein generelles Verbot von Erbverträgen aus. Gerade diese Besonderheiten des italienischen Erbrechts haben in der Vergangenheit zu einer Vielzahl von Reformdiskussionen Anlass gegeben, die allerdings seit der Familienrechtsreform des Jahres 1975 zu keiner grundlegenden Reform des Erbrechts geführt haben. Nun sind derzeit zwar keine konkreten Pläne für eine Novellierung des italienischen Erbrechts bekannt, die Diskussionen um eine Einschränkung des Ehegattenerbrechts, eine Neugestaltung des Pflichtteilsrechts und eine Abschaffung des Erbvertrags halten aber an, ${ }^{80}$ sodass sich der Gesetzgeber dem immer drängender werdenden Wunsch nach einer Modernisierung nicht auf unbestimmte Zeit wird entziehen können.
70. Ferri (Fn 34) $252 \mathrm{f}$.

71. Cass civ 15.6.2006, Nr. 13804, Giustizia civile massimario 2006, 1608.

72. Diese erfüllt den Zweck den Schenkungsempfänger und Vermächtnisnehmer Einblick in den Umfang der Erbschaft zu geben und sie vor unberechtigten Ansprüchen der Pflichtteilsberechtigten zu schützen. Siehe Franco (Fn 38) 1395; Bonilini, Manuale (Fn 17) 197.

73. Mengoni (Fn 33) 242 ff; Ferri (Fn 34) 199 ff; Franco (Fn 38) 1405. In der Rsp: LG Lucca 2.7.2007, Nr. 864, Giurisprudenza di merito 2008, 738.

74. Franco (Fn 38) $1386 \mathrm{ff}$.

75. Kontrovers diskutiert wird bezüglich der Herausgabe beweglicher Sachen, ob eine analoge Anwendung von Artikel 560 CC möglich ist (Mengoni (Fn 33) 291), oder ein Wahlrecht des Schenkungsempfängers oder Vermächtnisnehmers besteht, die Sache oder den Gegenwert herauszugeben (Bianca (Fn 34) 219).

76. Das Gesetz Nr. 80/2005 hat allerdings bewirkt, dass die Belastungen und Hypotheken wirksam bleiben, 'wenn die Kürzung erst nach mehr als zwanzig Jahren ab der Eintragung der Schenkung verlangt worden ist' (Art. 561 CC). Auch hier muss allerdings ein wertmäßiger Ausgleich stattfinden, wenn 'der Anspruch innerhalb von zehn Jahren ab Eröffnung der Erbfolge geltend gemacht worden ist'. Kritisch dazu Romano (Fn 49) $684 \mathrm{ff}$.
77. Der Ehegatte oder eingetragene Partner sowie die Verwandten in gerader Linie des Schenkers können der Schenkung durch außergerichtliche Erklärung widersprechen und somit den Ablauf der vorgesehenen Frist hemmen (Art. 563 Abs. 4 CC); vgl. allerdings die besonderen Fristen für die Eintragung der Kürzungsklage (Art. 2652 Nr. 8 CC, 2690 Nr. 5 CC).

78. Diese zeitliche Beschränkung wurde zum Schutz des Dritterwerbers sowie zum allgemeinen Schutz des Rechtsverkehrs durch die L 80/2005 eingeführt. Siehe Bonilini, Manuale (FN 17) 203. Kürzere Fristen gelten im Grundbuchssystem, siehe Eccher (FN 71) 689 Fn 433.

79. Christandl, Der italienisch-österreichische Erbfall (Fn 40) 213. In der Rsp: Cass sez un 25.10.2004, Nr. 20644 Giurisprudenza italiana 2005, 1605. Eine ausführliche Schilderung der langjährigen Debatte über den dies a quo liefert Franco (Fn 38) $1415 \mathrm{ff}$.

80. leva, Appunti per un'ipotesi di revisione del divieto dei patti successori, Rivista del notariato 2018, 1 ff.; leva, La tutela dei legittimari: oscillazioni fra contrapposte istanze sociali, Rivista del notariato 2017, 207 ff.; Lipari, Prospettive della libertà di disposizione ereditaria, Rivista trimestrale di diritto e procedura civile 2016, 799 ff.; Bonilini, Sulla novellazione delle norme relative alla successione necessaria, Ricerche giuridiche 2013, $283 \mathrm{ff}$. 